University of Wollongong

Research Online

Faculty of Engineering - Papers (Archive)

Faculty of Engineering and Information

Sciences

January 2006

\title{
A robust motion design technique for flexible-jointed manipulation systems
}

G. Alici

University of Wollongong, gursel@uow.edu.au

S. Kapucu

Gaziantep University, Turkey

S. Baysec

Gaziantep University, Turkey

Follow this and additional works at: https://ro.uow.edu.au/engpapers

Part of the Engineering Commons

https://ro.uow.edu.au/engpapers/321

\section{Recommended Citation}

Alici, G.; Kapucu, S.; and Baysec, S.: A robust motion design technique for flexible-jointed manipulation systems 2006.

https://ro.uow.edu.au/engpapers/321

Research Online is the open access institutional repository for the University of Wollongong. For further information contact the UOW Library: research-pubs@uow.edu.au 


\section{A robust motion design technique for flexible-jointed manipulation systems Gürsel Alıc1*, Sadettin Kapucu** and Sedat Bayseç**}

(Received in Final Form: February 27, 2005)

\begin{abstract}
SUMMARY
This paper presents a robust input shaping technique that significantly reduces (almost eliminates) the residual vibration of manipulation systems typified by a flexiblejointed robot manipulator. The technique consists of two stages. In the first stage, a ramp function is superimposed onto the main trajectory to be preshaped. In the second stage, the outcome of the first stage is convolved with a sequence of two impulses. The robustness of the technique to the uncertainties in the system natural frequency and damping ratio are quantified through simulation and experimental evaluation. Simulation and experimental results demonstrate that the technique is not only effective in reducing the residual vibrations, but also it is robust to the uncertainties of as high as $\mp 35 \%$ from the ideal value of the system natural frequency. Further, it has been found that the proposed input shaping technique is insensitive to the uncertainties in the damping ratio. The results allow us to suggest that the proposed technique is versatile and robust enough to apply it to the motion design of any flexible-jointed manipulation system making a point-to-point motion.
\end{abstract}

KEYWORDS: Motion design/input shaping; Flexiblejointed robots; Robustness; Residual vibration elimination.

\section{INTRODUCTION}

Command preshaping or input shaping or motion design can be considered as a feedforward control approach modifying a specified motion profile such that the system gives a desired output such as a minimum amplitude vibration when it completes its motion. As a challenging motion design problem for manipulation systems, it has attracted the attention of many researchers. ${ }^{1-14}$ Of these studies, Aspinwall ${ }^{3}$ has used the Fourier expression of a sine series for the forcing function to obtain a vibration free motion of a flexible system. Sahinkaya ${ }^{8}$ describes a method for input shaping to produce desired output by inverse dynamics using third order polynomials. The robustness of the method has been evaluated through the simulation results, and a significant improvement in the parameter errors has been

\footnotetext{
* Corresponding author: E-mail: gursel@uow.edu.au

* University of Wollongong, School of Mechanical, Materials and Mechatronics Engineering, NSW 2522, Wollongong (Australia).

** Gaziantep University, Faculty of Engineering, Department of Mechanical Engineering, TR-27310, Gaziantep (Turkey).
}

shown. Meckl and Seering ${ }^{11}$ have developed a set of shaped force profiles to reduce residual vibration of velocity limited dynamic systems. The force profiles are constructed from a versine (1-cosine) function, which has no discontinuity in slope at the beginning and end, and its harmonics with coefficients are chosen to minimise the energy of the resulting function near the system natural frequency. This is accomplished by employing an optimization technique. Other command shaping techniques include selecting a limited number of acceleration inputs to reduce the residual vibration. Cuciouo et al. ${ }^{4}$ have presented an acceleration trajectory varying only by equal steps of equal duration and applied it to two mechanical devices for testing the effectiveness of their method. Mimmi and Pennachi ${ }^{6}$ have proposed an input shaping strategy based on two types of acceleration inputs; the first one is a constant acceleration input with a different time interval, and the second one is a trapezoidal acceleration input. Alici et al. ${ }^{2}$ have proposed an acceleration trajectory consisting of a cycloid, a constant velocity, and a cycloid. They have applied it to the swing free transportation of simply suspended objects with a robot manipulator.

Input preshaping based on convolving the desired motion profile with a sequence of impulses has found a great application in reducing residual vibrations in computer-controlled machines. ${ }^{9}, 11,12$ This method requires perfect knowledge of the system parameters such as natural frequency and damping ratio to minimize the residual vibration. The more impulses are used, the more robust the system becomes to system parameter variations, but with a penalty of greater travel time to complete the move. Many researchers have implemented this method. Of these, Murpy and Watanable ${ }^{7}$ have reported on a digital shaping filter based on convolving a sequence of impulses to reduce residual vibration. Alici et al. ${ }^{1,2,10}$ have proposed a methodology based on a ramp motion profile superimposed onto a cycloid for input shaping in order to compare its efficacy and robustness to those of the input shaping method proposed by Singer and Seering. ${ }^{9}$ Later, they applied both methods successively to reduce the swing of a simply suspended object transported with a robot manipulator and have concluded that it could be applied to a multi-mode system, and it is more robust to the uncertainties of the system natural frequency without increasing the travelling time.

The technique we present in this study is the extension of that input shaping method to the residual vibration elimination of flexible-jointed robot manipulators. However, it should be emphasized that the problem considered here 
differs from the motion design problem in our previous work in two aspects; firstly, the system under consideration is a flexible jointed robot manipulator, and most importantly, secondly, the system is a damped system. We call this technique a hybrid input shaping technique since it is based on the subsequent application of two input shaping methods. These methods are (i) superimposing a ramp function onto a main desired function, and (ii) convolving a sequence of impulses with a desired input to generate a shaped input. Theoretical background, new simulation and experimental results indicating the effectiveness of this technique in reducing residual vibration are presented in this paper. We consider a trajectory based on a cycloidal motion, which is commonly used as a high-speed cam profile, continuous throughout one cycle, plus a ramp. The ramp function can be superimposed onto any mathematically described function in order to give an initial velocity to the system, which generates a vibration equal in magnitude, but out of phase to the vibration imposed by the main trajectory. The amplitude of the ramp and the cycloidal function are determined such that a total distance to be travelled and the transportation time are satisfied. Then the resulting trajectory is convolved with a sequence of two impulses to obtain a twice-shaped input. It is believed that this method reduces the vibration of manipulation systems with not exactly known dynamic parameters such as natural frequency and the damping ratio. The proposed technique has been implemented on a hydraulically driven Stanford type manipulator. A flexible jointed link is attached to one of its rotational joints having a direction of rotation perpendicular to the ground. The servo valve and actuator dynamics are considered in the simulations. It is assumed that the compliance existing in the transmission and structural elements does not cause considerable vibration of the manipulator endpoint during and at the end of the move. The simulation and experimental results indicate that a residual vibration free stop is obtainable at the end of a point-to-point move with a high degree of robustness to uncertainties in the system natural frequency and damping ratio by employing the proposed hybrid method.

\section{SYSTEM MODELING}

A schematic representation of a flexible jointed link connected to the hub of a rotary joint of the manipulator is shown in Figure 1. Servo valves control the position of the manipulator links in a closed loop fashion. The link attached to the hub has the inertia of $J$. The flexible jointed link with length $L$ carries a point mass $m$ at its tip. The manipulator flexibility, which is assumed to be due to the joint elasticity rather than the structural elasticity, is modeled with a torsional spring with a spring constant of $k .{ }^{15}$ For the sake of completeness, a damper with damping coefficient of $c$ is also added to the lumped parameter model of the flexiblejointed link. The angle between the hub and a horizontal line is $\theta$, and the angle of twist of the torsional spring is $\phi$. A mathematical model of the manipulator can be obtained by using the Lagrangian formulation. Neglecting Coulomb friction of the joint and assuming that the manipulator operates in the horizontal plane, the equations of motion of the flexible jointed system are derived as

$$
m L^{2} \ddot{\theta}+c_{h} \dot{\theta}+m L^{2} \ddot{\phi}=r\left(P_{1} A_{1}-P_{2} A_{2}\right)
$$

and

$$
\ddot{\theta}+\ddot{\phi}+\left(\frac{c}{m L^{2}}\right) \dot{\phi}+\left(\frac{k}{m L^{2}}\right) \phi=0
$$

where the pressures $P_{1}$ and $P_{2}$ in the actuator are functions of the supply pressure, the coefficient of leakage flow, flow gain of spool stage, area of the piston, actuator speed and spool displacement, ${ }^{11} A_{1}$ and $A_{2}$ are the cross sectional areas of the piston, and $c_{h}$ is the coefficient of viscous friction which is an inherent characteristics of a hydraulic system. It must be noted that for $c=0.0$, the equation of the motion of the rigid

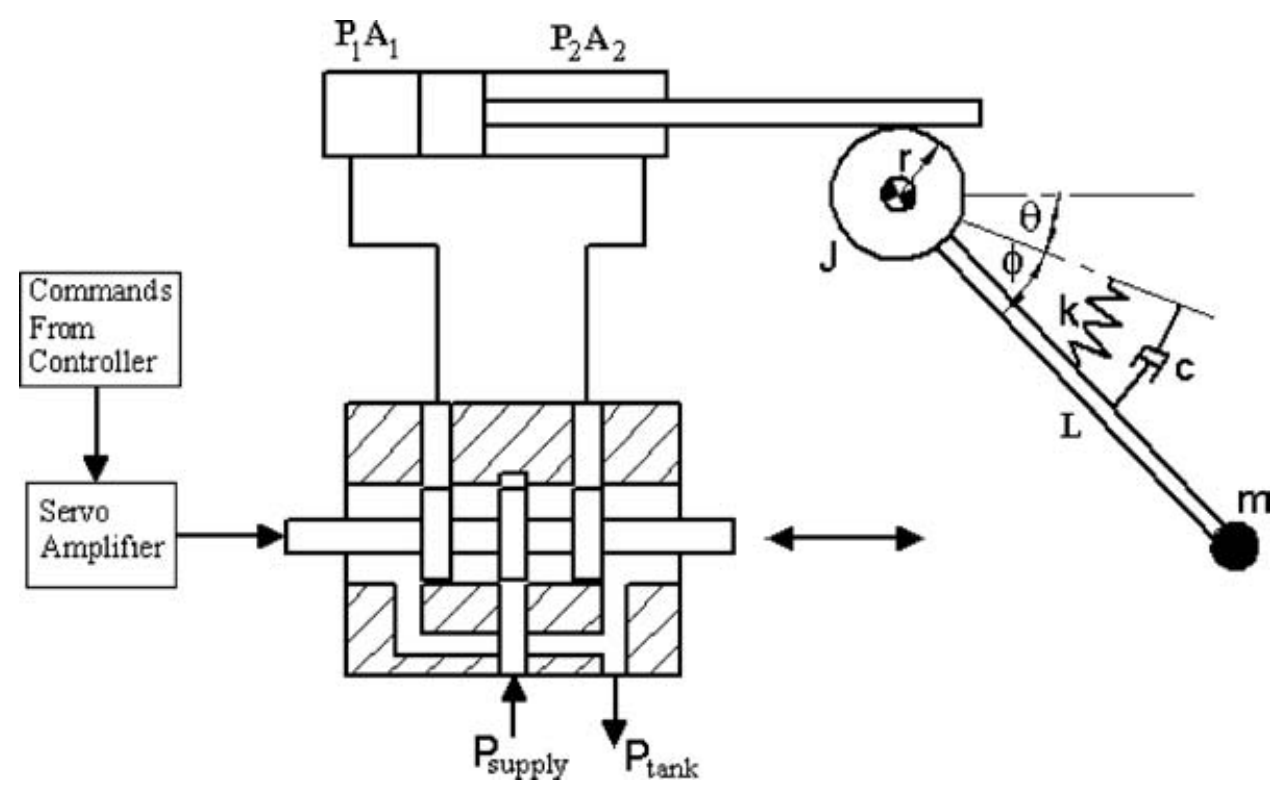

Fig. 1. Schematic representation of a single joint flexible link manipulator driven by a servo hydraulic system. 
link represents a simple periodic motion as if the rigid link is accelerating with $\ddot{\theta}$, which is the forcing function exciting the system. Depending on the form of $\ddot{\theta}$, the solution for Eq. 2 can be obtained. The problem here is to plan a reference input for the rotational joint of the manipulator such that the flexible jointed link moves from one point to another in the shortest possible time with a zero vibration at the completion of the motion.

\section{INPUT SHAPING FOR RESIDUAL VIBRATION REDUCTION}

The rotary link of the manipulator is desired to follow the input $\theta$. The hybrid input shaping technique consists of two stages; (i) superimposing a ramp function onto the specified trajectory, and (ii) the resulting two-piece trajectory is convolved with a sequence of two impulses to generate a shaped input. A cycloid is chosen as the desired trajectory to be preshaped. Note that the resulting trajectory is preshaped twice. The first stage neither limits nor increases the traveling time while the second stage increases the traveling time by a semi-period of the first mode of the system as presented below. Since each stage by itself can be employed to obtain a preshaped reference input, ${ }^{2}$ it is of use to present theoretical background for them.

\section{III.1. Superimposition based input shaping}

The reference input for the rotational link consists of two functions. The total distance to be covered from the beginning to the end of a move within a specified move time is the sum of the distances to be traveled by each of the two functions within the same period of time. By adjusting the excursion distance of each function, the vibration of the flexible joint link can be eliminated provided that the specified move time and the total distance are unchanged. Each component of the reference input for the rotational link creates oscillations of equal amplitude, out of phase such that they cancel each other and no vibration results. Cycloid-plus-ramp function motion profile is expressed as;

$$
\theta=\frac{\theta_{1}}{2 \pi}[2 R t-\sin (2 R t)]+\frac{\theta_{2}}{\tau} t
$$

where $\theta_{1}$ is the maximum excursion distance to be traveled by the cycloid motion profile, $\theta_{2}$ is the maximum excursion distance to be traveled by the ramp motion profile, $t$ is time into motion, $\tau$ is the traveling time, and $R=\pi / \tau$. For zero initial conditions and no damping, the equation of motion given by Eq. 2 is solved for the ramp and cycloid inputs defined by Eq. 3. In order to have no residual vibration after $t>\tau$, the distances $\theta_{1}$ and $\theta_{2}$ must be: $:^{2,10}$

$$
\theta_{1}=\theta\left[1-\left(\frac{\tau_{n}}{\tau}\right)^{2}\right], \quad \theta_{2}=\theta\left(\frac{\tau_{n}}{\tau}\right)^{2}
$$

where $\tau_{n}$ is the period of natural oscillation. For a very small damping ratio $\xi$, the response of the system will be very similar to that of an undamped system. ${ }^{16}$ This allows us to determine $\theta_{1}$ and $\theta_{2}$ from Eq. 4 .

\section{III.2. Convolution based input shaping}

This method, which involves convolving a sequence of impulses with the main desired trajectory, has been proposed by Singer and Seering. A full account of this method is given in reference [9]. It is based on the transient response of a second order system with the natural frequency of $\omega_{n}$, and the expected damping ratio of $\xi$ to an impulse input. The transient response $c(t)$ is expressed as:

$$
c(t)=\left[A \frac{\omega_{n}}{\sqrt{1-\varsigma^{2}}} e^{-\varsigma \omega_{n}\left(t-t_{o}\right)}\right] \sin \left[\omega_{n} \sqrt{1-\varsigma^{2}}\left(t-t_{o}\right)\right]
$$

where $A$ is the amplitude of the position impulse command, $t$ is time, $t_{o}$ is the time of the impulse input. Since any arbitrary function can be formed from a sequence of impulses, the impulse sequence can be used to reduce the vibration of dynamic systems under arbitrary trajectories. ${ }^{5}$ This superposition is accomplished by convolving any desired trajectory with a sequence of impulses in order to yield the shortest actual system input. This operation, therefore, becomes a prefilter for any input to be given to the system. The time penalty resulting from prefiltering the input equals to the length of the impulse sequence.

The amplitudes and the durations of the impulses are determined such that the system moves without vibration after the motion has ended. When a sequence of two impulses is used to reduce the residual vibration of the system under consideration here, the durations and amplitudes of the impulses are found as: ${ }^{10}$

$$
\begin{array}{ll}
t_{1}=0, & t_{2}=\frac{\pi}{\omega_{n} \sqrt{1-\xi^{2}}}, \\
A_{1}=\frac{1}{K+1}, & A_{2}=\frac{K}{K+1},
\end{array}
$$

where $A_{1}$ and $A_{2}$ are the amplitude of the position impulse command, $t_{1}$ and $t_{2}$ are time, $K=e^{-\xi \pi / \sqrt{1-\xi^{2}}}$. Note that the amplitudes of the two impulses are normalised so that they sum to unity. These are the shortest duration sequences that eliminate residual vibrations, assuming that only positive amplitudes are used. It has been reported ${ }^{9}$ that the response is extremely sensitive to uncertainties in the natural frequency and damping ratio of the system. Some additional constraints can be added to increase robustness against the uncertainties. This necessitates the use of more than two impulses with a penalty of increasing the move time, which is not very desirable. When the system has no damping, the time $t_{2}$ of the second impulse is a half of the period of natural oscillation of the system and the duration of the motion is lengthened by $t_{2}$.

\section{III.3. Proposed hybrid input shaping technique}

This method is based on the two methods just presented above. Both of these methods are sensitive to uncertainties in the natural frequency and damping ratio of the system when they are individually applied. The beneficial features of these methods are that while the former does not increase the traveling duration, and the latter can be applied to any 

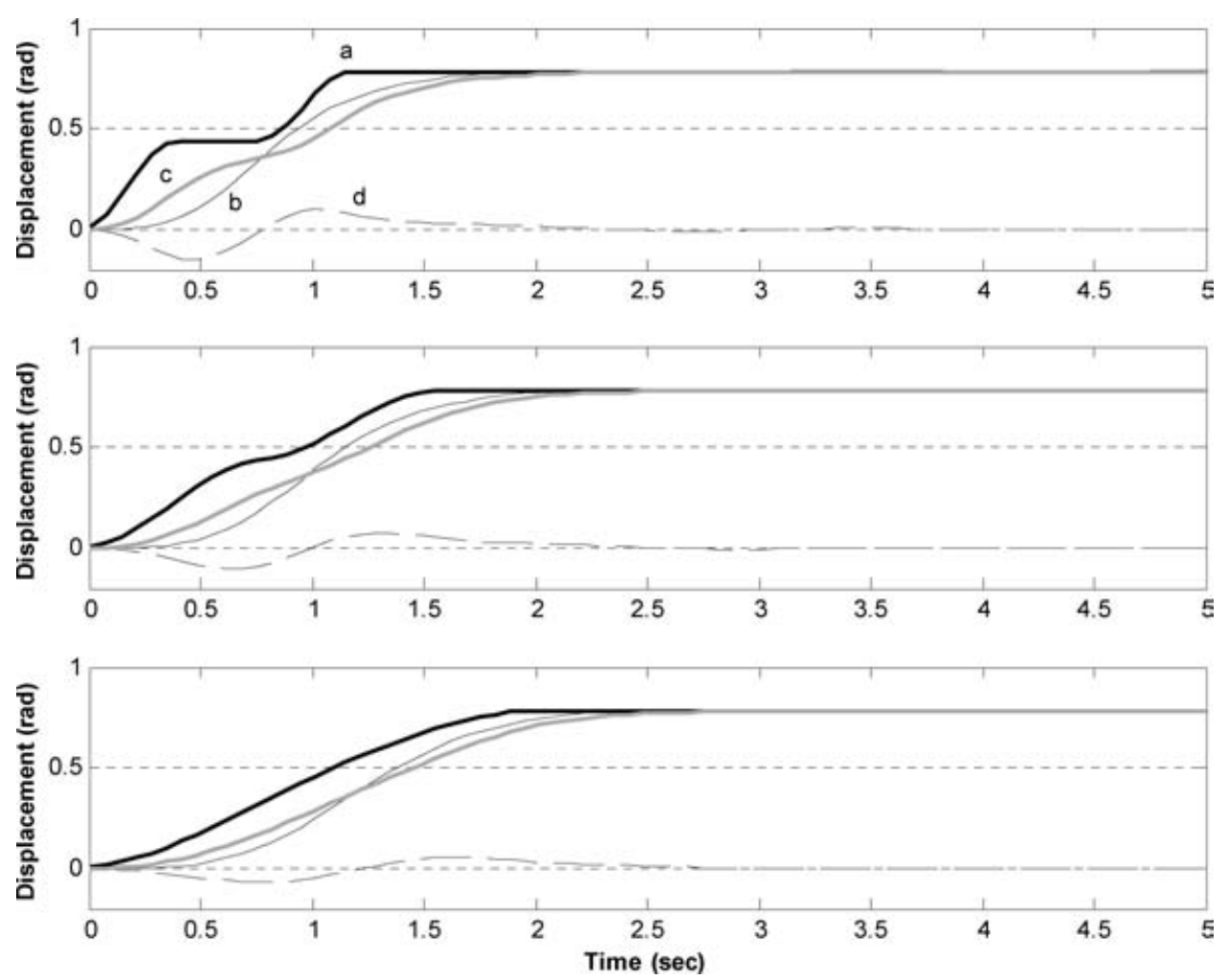

Fig. 2. Simulation results from the hybrid input shaping technique for a traveling distance of $\theta=\pi / 4 \mathrm{rad}$. $\theta_{1}$ and $\theta_{2}$ are arbitrarily taken as $\pi / 8$ and $\pi / 8$ radians, respectively, and for the traveling times of $\tau_{t}=1.5 \pi / \omega_{n}$ seconds (top plot), $\tau_{t}=2.0 \pi / \omega_{n}$ seconds (middle plot), and $\tau_{t}=2.5 \pi / \omega_{n}$ seconds (bottom plot).
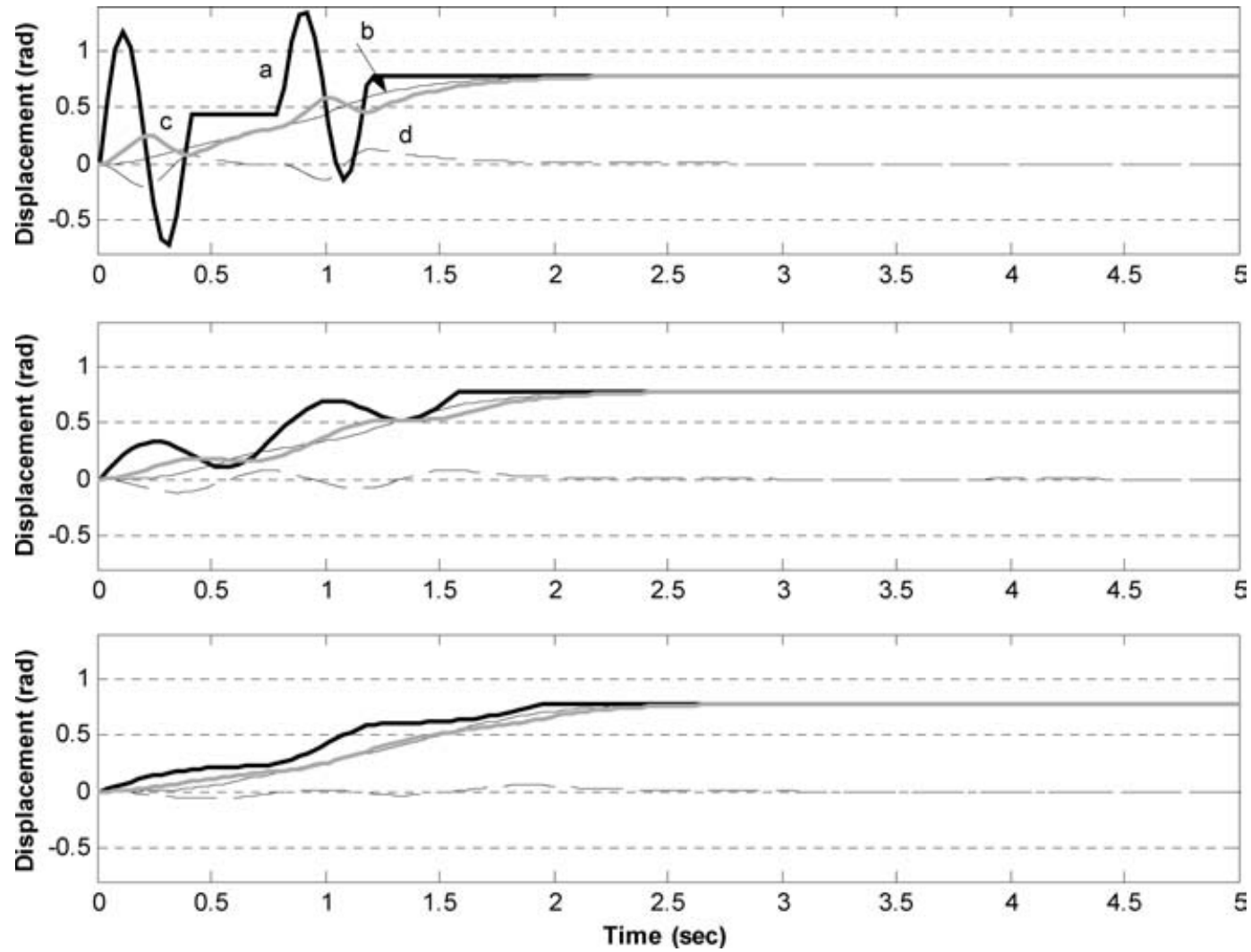

Fig. 3. Simulation results from the hybrid input shaping technique for a total traveling distance of $\theta=\pi / 4 \mathrm{rad}$. $\theta_{1}$ and $\theta_{2}$ are determined from Eq. 4. The plot (a) is for $\tau_{t}=1.5 \pi / \omega_{n}$ seconds, $\theta_{1}=-11.781 \mathrm{rad}$ and $\theta_{2}=12.566 \mathrm{rad}$. The plot (b) is for $\tau_{t}=2.0 \pi / \omega_{n}$ seconds, $\theta_{1}=-2.356 \mathrm{rad}$ and $\theta_{2}=3.141 \mathrm{rad}$. The plot (c) is for $\tau_{t}=2.5 \pi / \omega_{n}$ seconds. $\theta_{1}=-0.611 \mathrm{rad}$ and $\theta_{2}=1.396 \mathrm{rad}$. 


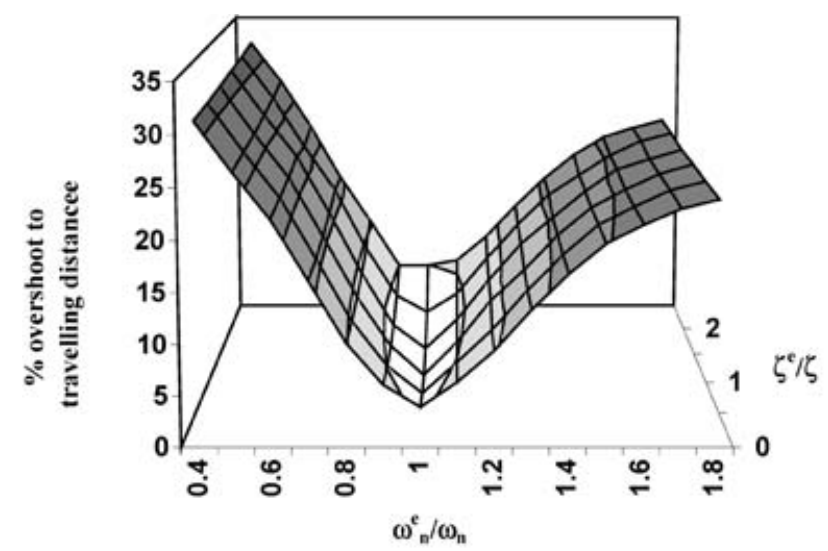

(a)

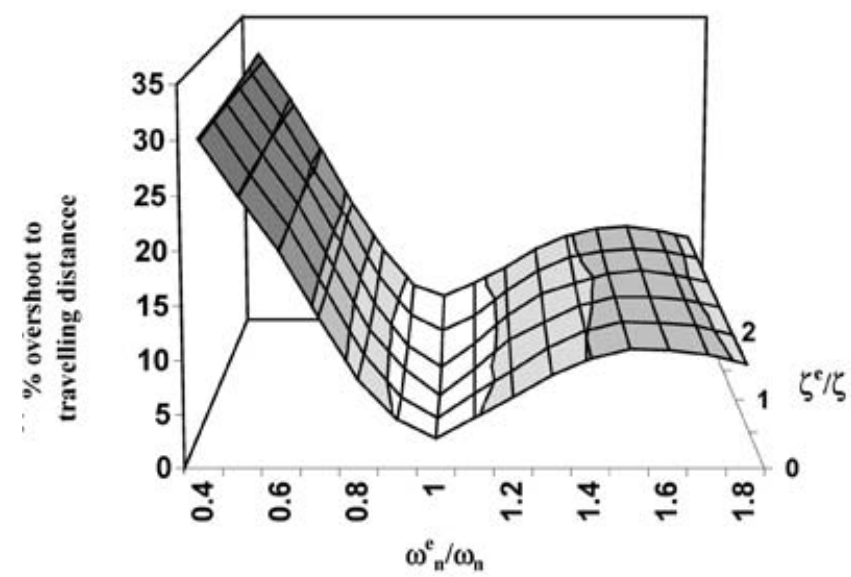

(b)

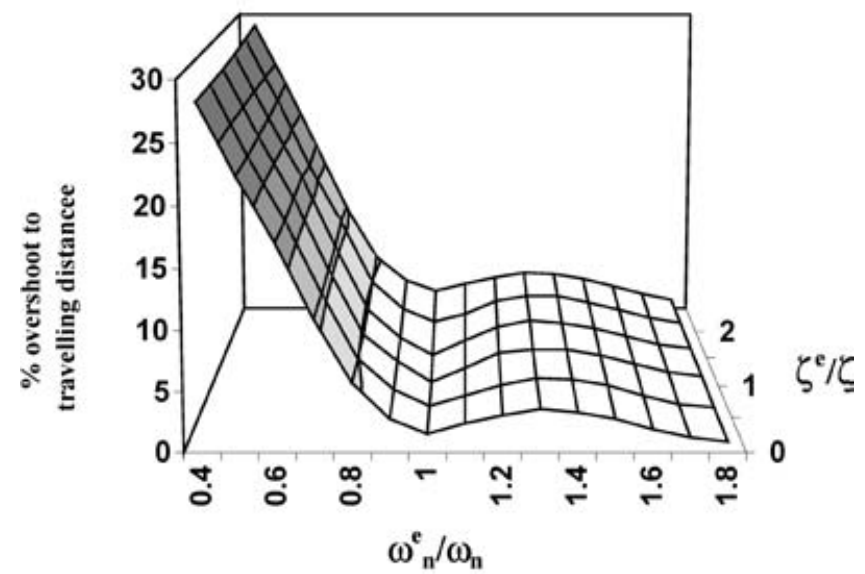

(c)

Fig. 4. Simulated sensitivity of the system to uncertainties in the system natural frequency and damping ratio for a total traveling distance of $\theta=\pi / 4 \mathrm{rad}$. $\theta_{1}$ and $\theta_{2}$ are arbitrarily taken as $\pi / 8$ and $\pi / 8$ radians, respectively. The plot (a) is for $\tau_{t}=1.5 \pi / \omega_{n}$ seconds, the plot (b) is for $\tau_{t}=2.0 \pi / \omega_{n}$ seconds, and the plot (c) is for $\tau_{t}=2.5 \pi / \omega_{n}$ seconds.

trajectory. The hybrid input shaping method we propose not only contains their beneficial features, but also is robust to the natural frequency and damping ratio uncertainties.

It is important to note that the preshaped command function does not contain impulses once the convolution is performed. The convolution of any desired command function with some impulse sequence results in a trajectory

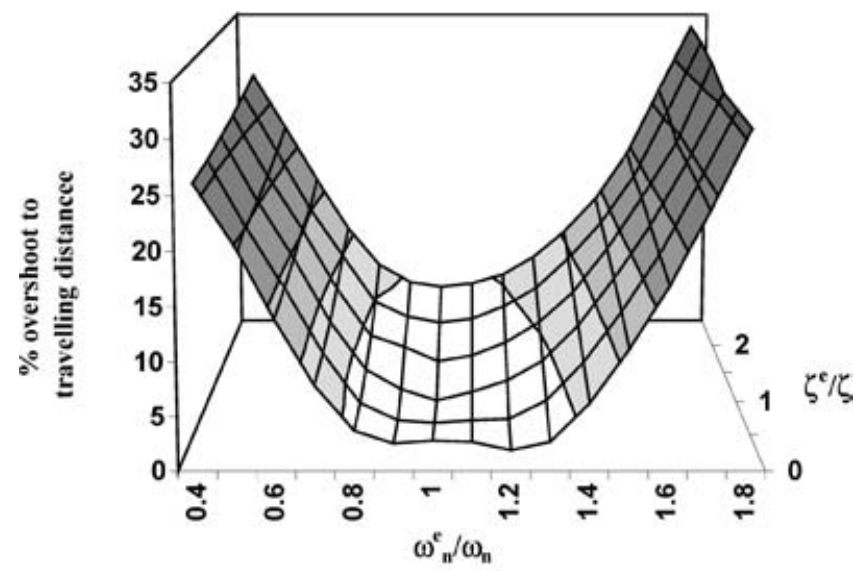

(a)

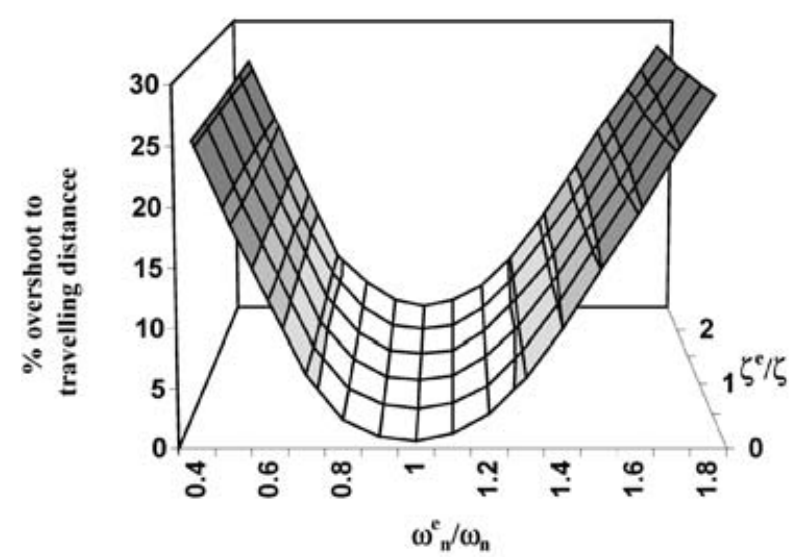

(b)

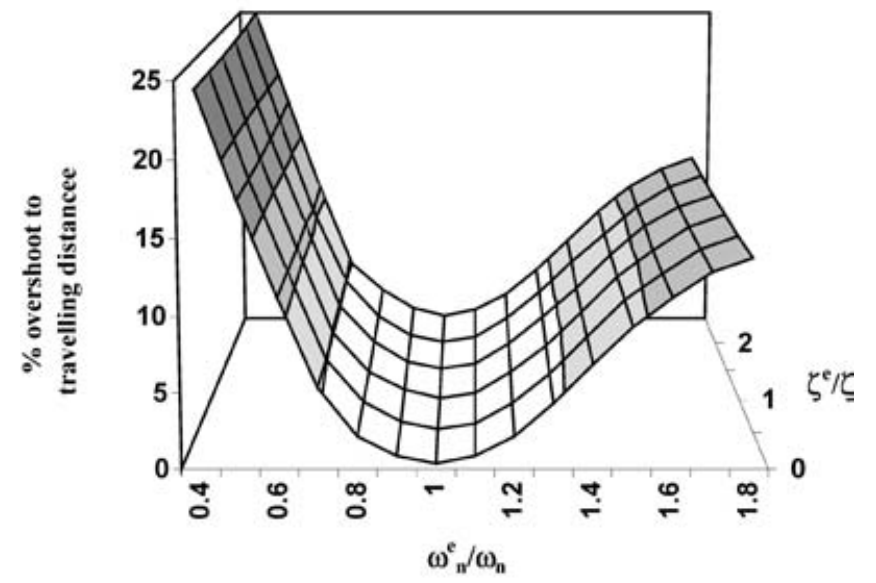

(c)

Fig. 5. Simulated sensitivity of the system to uncertainties in the system natural frequency and damping ratio for a total traveling distance of $\theta=\pi / 4 \mathrm{rad}$. $\theta_{1}$ and $\theta_{2}$ are determined from Eq. 4 . The plot (a) is for $\tau_{t}=1.5 \pi / \omega_{n}$ seconds, $\theta_{1}=-11.781 \mathrm{rad}$ and $\theta_{2}=$ $12.566 \mathrm{rad}$. The plot (b) is for $\tau_{t}=2.0 \pi / \omega_{n}$ seconds, $\theta_{1}=$ $-2.356 \mathrm{rad}$ and $\theta_{2}=3.141 \mathrm{rad}$. The plot (c) is for $\tau_{t}=2.5 \pi / \omega_{n}$ seconds. $\theta_{1}=-0.611 \mathrm{rad}$ and $\theta_{2}=1.396 \mathrm{rad}$.

that has the same vibration reducing effects as the impulse sequence. ${ }^{9}$ For a specified traveling time and displacement, hybrid-shaping technique is implemented as follows;

(i) Traveling time for the cycloid is calculated from $\tau_{1}=\tau_{t}-\tau_{2}$ in order to satisfy the total traveling time $\tau_{t}$ 


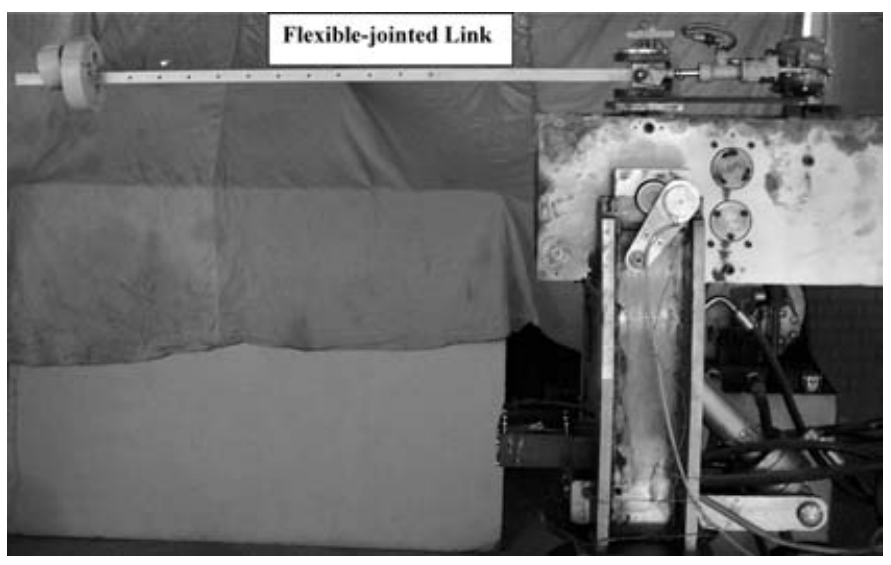

Fig. 6. Experimental set-up: A Stanford type manipulator with a flexible jointed link.

(ii) The distances $\theta_{1}$ and $\theta_{2}$ for a cycloid and a ramp function are calculated from Eq. 4,

(iii) The resulting trajectory is convolved with the twoimpulse sequence defined by Eq. 6 .

\section{NUMERICAL ANALYSIS}

The differential equation of motion given by Eqs. 1 and 2 are solved numerically by using the preshaped commands consisting of a cycloid + a ramp convolved with a sequence of two impulses. The servo valve and actuator dynamics are considered in the simulations. Two sets of simulations are performed for three different traveling times, which are $\tau_{t}=1.5 \pi / \omega_{n}, \tau_{t}=2 \pi / \omega_{n}$ and $\tau_{t}=2.5 \pi / \omega_{n}$. In the first set, $\theta_{1}$ and $\theta_{2}$ excursions of the ramp and cycloid are taken arbitrarily without considering Eq. 4 . In the second set, they are calculated from Eq. 4. The numerical values used in both sets are $\omega_{n}=4 \mathrm{rad} / \mathrm{sec}, \xi=0.095$ (9.5\% of the critical damping of the flexible-jointed link) and $\theta=\pi / 4$ radians. Eqs. 1 and 2 are solved for the hybrid input shaping, using Runge-Kutta numerical integration algorithms. Figure 2 shows the simulation results when the distances $\theta_{1}$ and $\theta_{2}$ are chosen without considering Eq. 4. Figure 3 shows the simulation results with the distances $\theta_{1}$ and $\theta_{2}$ determined from Eq. 4. In the simulation results provided in Figures 2 and 3 , the plot indicated with ' $a$ ' is the preshaped command, the plot indicated with ' $b$ ' is the input transmitted to the flexible-jointed link through the dynamic model of the servo valve and hydraulic actuator, the plot indicated with ' $c$ ' is the response of the link, and the plot indicated with ' $d$ ' is the vibration of the link. The plot ' $d$ ' is obtained from the difference between ' $b$ ' and ' $c$ '. Note that there is almost no residual vibration when $t>\tau$, and that there is a significant time delay in the simulated response of the manipulator and therefore in the simulated vibration. This time delay could be due to the servovalve and actuator dynamics considered in the simulations. The same time delay can also be seen in the experimental results given in Section V.

To evaluate the sensitivity of the system to the uncertainties in the system natural frequency and damping ratio, the maximum amplitude of the residual vibration (as a percentage of the total traveling displacement) for $\theta=\pi / 4$ radians and for the ranges of $0.4 \leq \omega_{n}^{e} / \omega_{n} \leq 1.8$ and $0 \leq \xi^{e} / \xi \leq 5$ is plotted against $\omega_{n}^{e} / \omega_{n}$ and $\xi^{e} / \xi$ in Figures 4 and 5. $\omega_{n}=4 \mathrm{rad} /$ sec and $\xi=0.095$ indicate the actual natural and damping ratio of the system considered in the input shaping procedure, respectively. $\omega_{n}^{e}$ and $\xi^{e}$ are the expected natural frequency and damping ratio, respectively. The delay due to the impulse sequence is $t_{2}=\pi / \omega_{n}$ seconds. In the simulation results depicted in Figures 4 and 5, the region of the plots denoted with white color indicates the uncertainty boundaries for a residual vibration less than $5 \%$ of the overshoot to the traveling distance. When the results in Figures 4 and 5 are compared to each other, the robustness results based on calculating the ramp and cycloidal excursions, $\theta_{1}$ and $\theta_{2}$, from Eq. 4 are better than those of the arbitrarily selected ones. However, both simulation results demonstrate that the hybrid input shaping method is quite robust to uncertainties in the natural frequency and damping ratio of the system. If the amplitude of the residual vibration for a dynamic system is less than $5 \%$, it is widely accepted that the system is in a vibration-free state after following a trajectory. ${ }^{10}$

\section{EXPERIMENTAL EVALUATION AND DISCUSSION OF RESULTS}

The experimental set-up is depicted in Figure 6. It consists of a hydraulically actuated manipulator of Stanford type, a conductive servo potentiometer to measure the oscillation of the flexible jointed link, and hardware to control, command the manipulator, and read the potentiometer output. A flexible jointed link is attached to its rotational joint moving perpendicular to the horizontal plane, and its other joints are fixed. The manipulator links are controlled by Bosch servo valves of 0811404028 type in a closed loop fashion, using the control strategy shown in Figure 7.

The object of the experimental work has been to illustrate the effectiveness of the input shaping technique in minimizing the amplitude of the residual vibration of the flexible-jointed link. Experiments have been conducted with the same parameter as described in previous section and the results are depicted in Figures 8, and 9. These results correspond to the simulation results presented in Figures 2 and 3 , respectively. When the corresponding figures are compared to each, it will be noticed that there is a close correspondence between the simulation and experimental

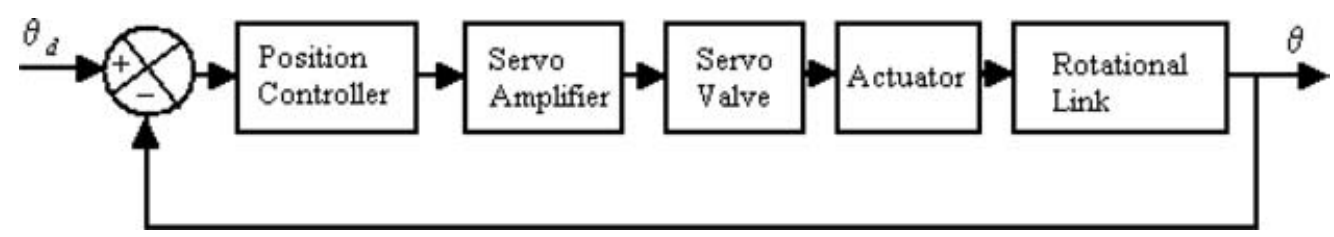

Fig. 7. Control strategy for the position control of the rotational link of the manipulator shown in Figure 6. 

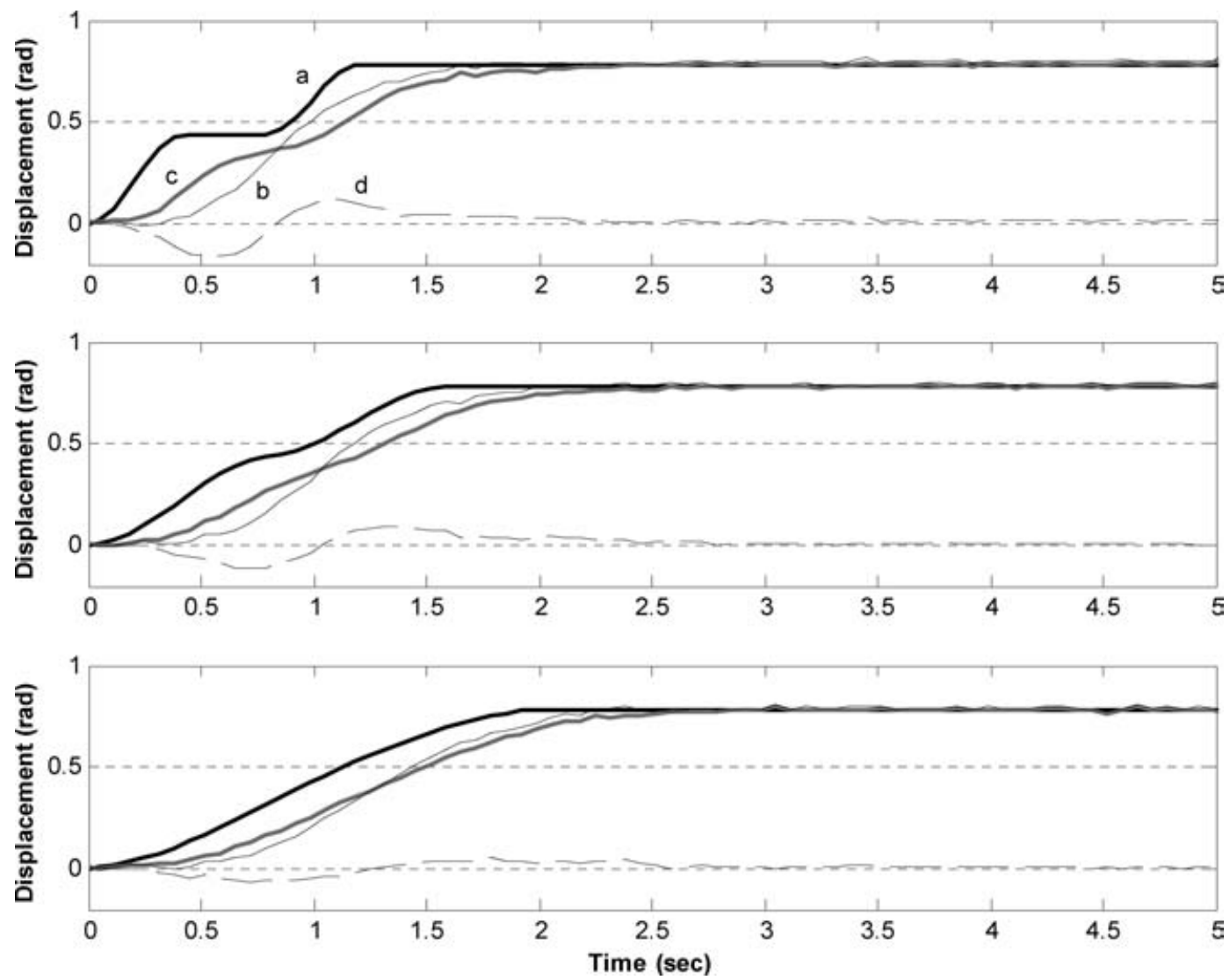

Fig. 8. Experimental results from the hybrid input shaping technique for a traveling distance of $\theta=\pi / 4 \mathrm{rad}$. $\theta_{1}$ and $\theta_{2}$ are arbitrarily taken as $\pi / 8$ and $\pi / 8$ radians, respectively, and for the traveling times of (a), $\tau_{t}=1.5 \pi / \omega_{n}$ seconds, (b) $\tau_{t}=2.0 \pi / \omega_{n}$ seconds, and (c) $\tau_{t}=$ $2.5 \pi / \omega_{n}$ seconds.
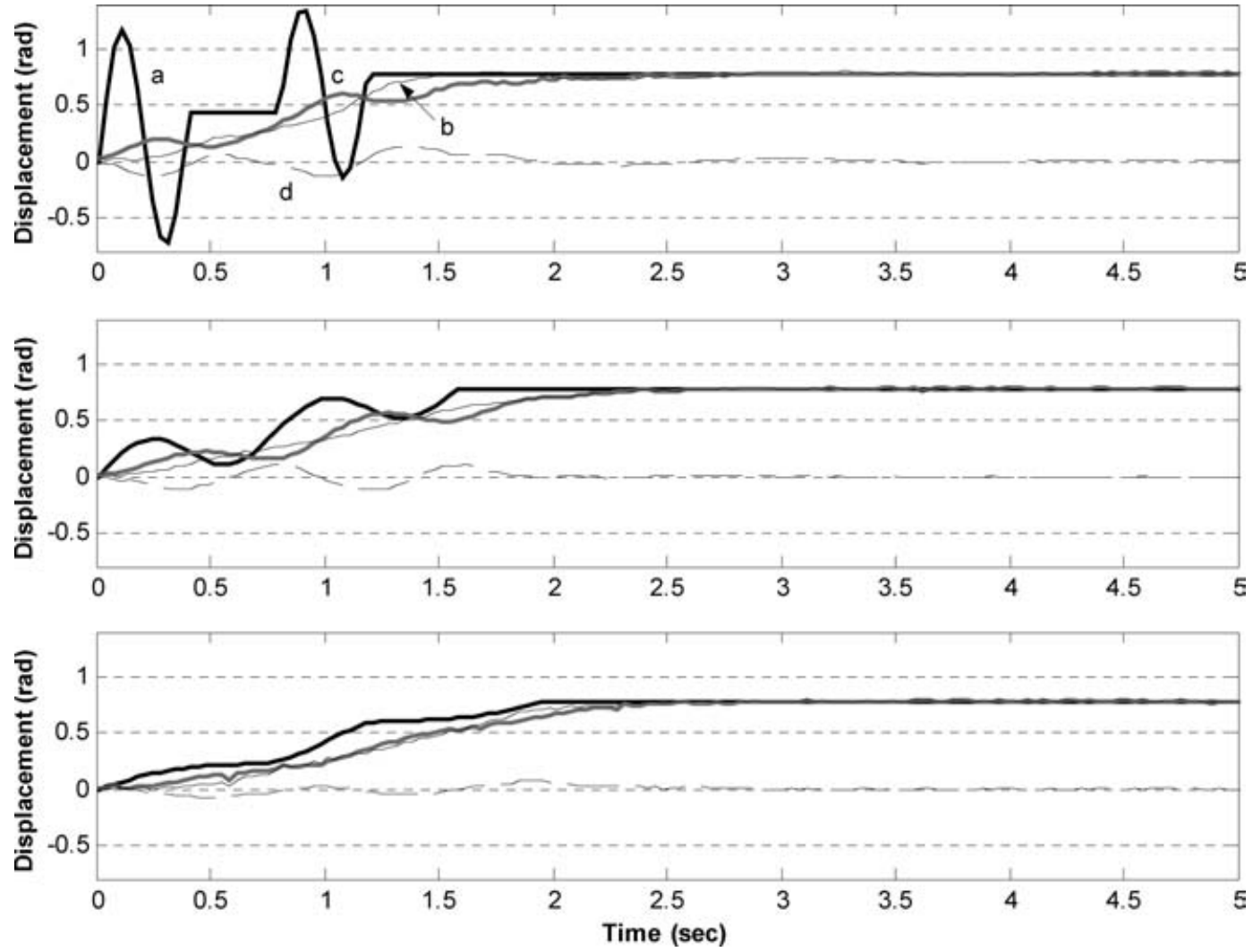

Fig. 9. Experimental results from the hybrid input shaping technique for a total traveling distance of $\theta=\pi / 4 \mathrm{rad}$. $\theta_{1}$ and $\theta_{2}$ are determined from Eq. 4. The plot (a) is for $\tau_{t}=1.5 \pi / \omega_{n}$ seconds, $\theta_{1}=-11.781 \mathrm{rad}$ and $\theta_{2}=12.566 \mathrm{rad}$. The plot (b) is for $\tau_{t}=2.0 \pi / \omega_{n}$ seconds, $\theta_{1}=-2.356 \mathrm{rad}$ and $\theta_{2}=3.141 \mathrm{rad}$. The plot (c) is for $\tau_{t}=2.5 \pi / \omega_{n}$ seconds. $\theta_{1}=-0.611 \mathrm{rad}$ and $\theta_{2}=1.396 \mathrm{rad}$. 


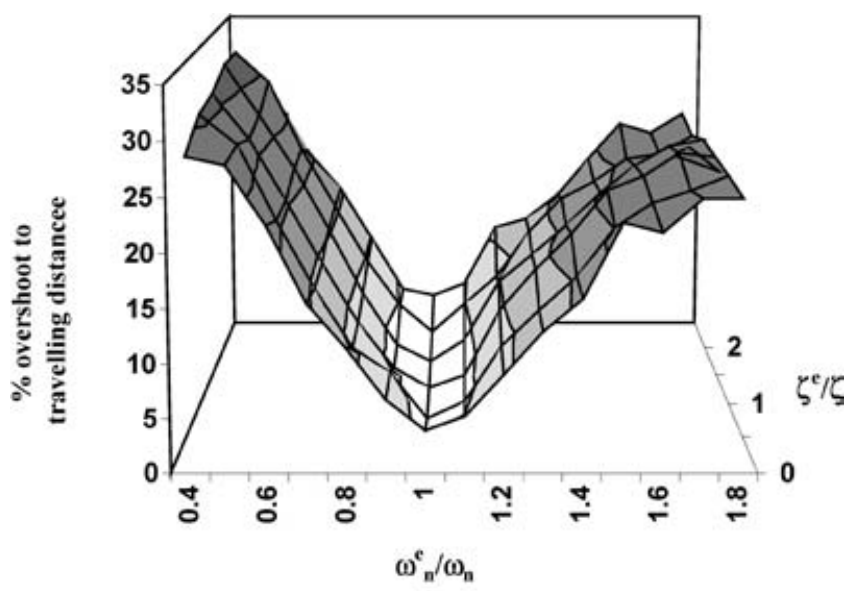

(a)

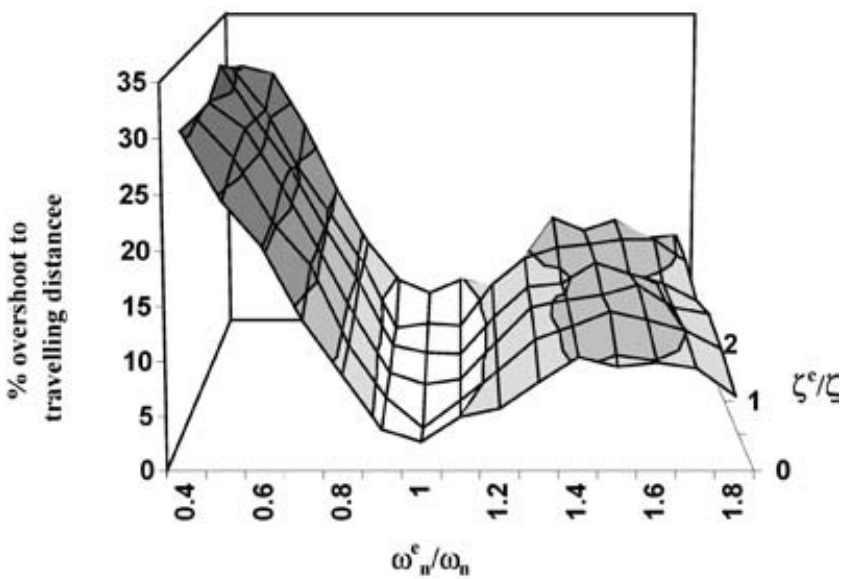

(b)

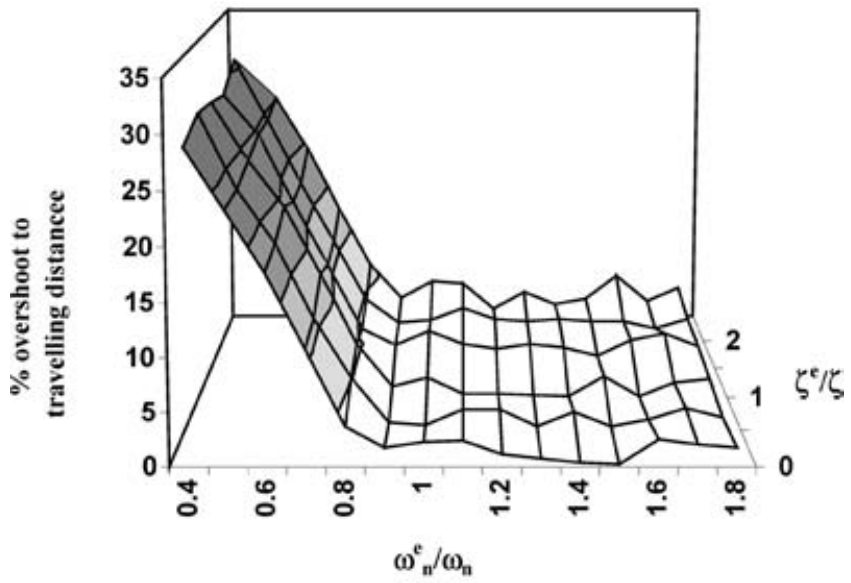

(c)

Fig. 10. Experimental sensitivity of the system to uncertainties in the system natural frequency and damping ratio for a total traveling distance of $\theta=\pi / 4 \mathrm{rad}$. $\theta_{1}$ and $\theta_{2}$ are arbitrarily taken as $\pi / 8$ and $\pi / 8$ radians, respectively. The plot (a) is for $\tau_{t}=1.5 \pi / \omega_{n}$ seconds, the plot (b) is for $\tau_{t}=2.0 \pi / \omega_{n}$ seconds, and the plot (c) is for $\tau_{t}=2.5 \pi / \omega_{n}$ seconds.

results. This suggests that the proposed input shaping technique performs well on a real system. In the results shown in Figures 8 and 9, the plot indicated with ' $a$ ' is the preshaped command, the plot indicated with ' $b$ ' is the input transmitted to the flexible-jointed link through the dynamic model of the servo valve and hydraulic actuator, the plot indicated with

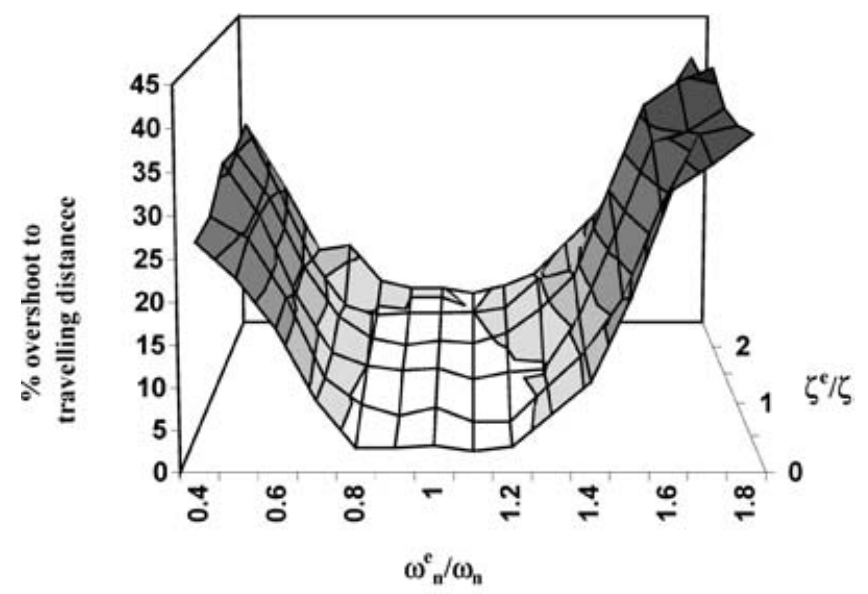

(a)

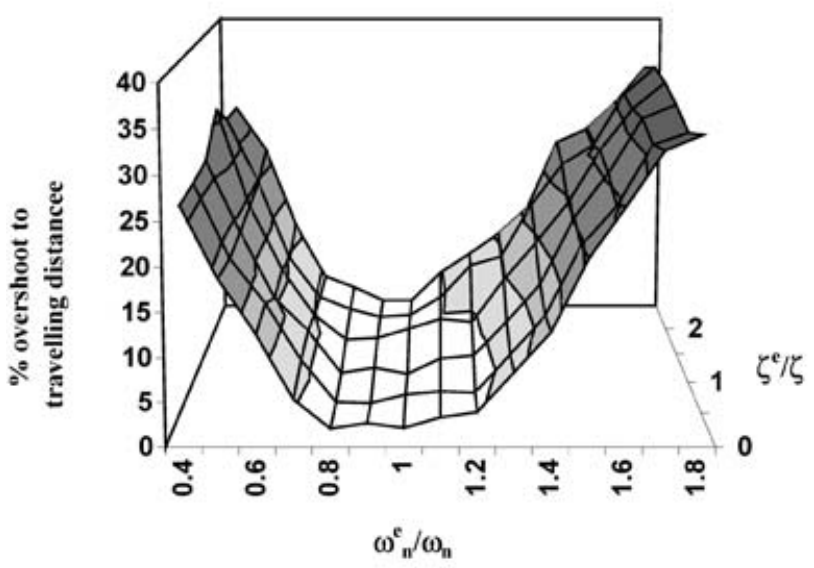

(b)

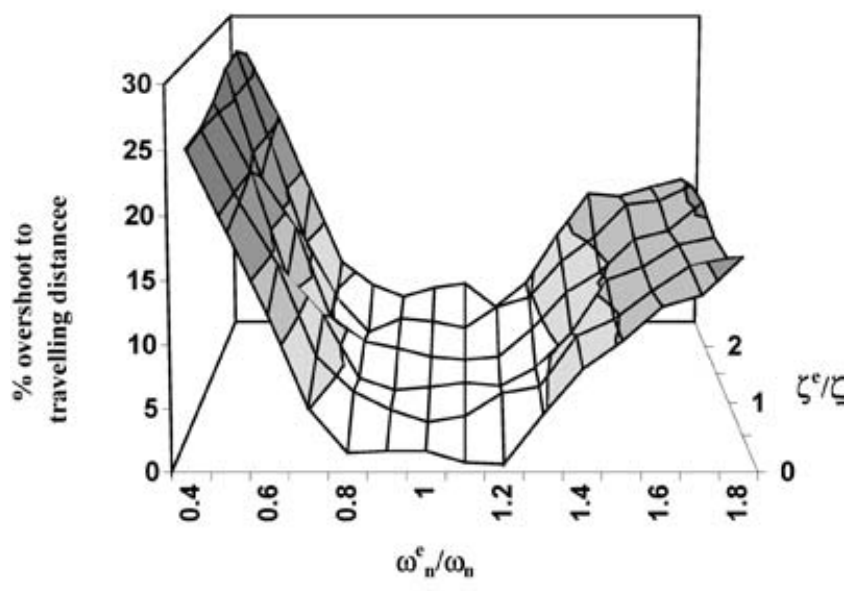

(c)

Fig. 11. Experimental sensitivity of the system to uncertainties in the system natural frequency and damping ratio for a total traveling distance of $\theta=\pi / 4 \mathrm{rad} . \theta_{1}$ and $\theta_{2}$ are determined from Eq. 4 . The plot (a) is for $\tau_{t}=1.5 \pi / \omega_{n}$ seconds, $\theta_{1}=-11.781 \mathrm{rad}$ and $\theta_{2}=12.566 \mathrm{rad}$. The plot (b) is for $\tau_{t}=2.0 \pi / \omega_{n}$ seconds, $\theta_{1}=$ $-2.356 \mathrm{rad}$ and $\theta_{2}=3.141 \mathrm{rad}$. The plot (c) is for $\tau_{t}=2.5 \pi / \omega_{n}$ seconds. $\theta_{1}=-0.611 \mathrm{rad}$ and $\theta_{2}=1.396 \mathrm{rad}$.

' $c$ ' is the response of the link, and the plot indicated with ' $d$ ' is the vibration of the link. The plot ' $d$ ' is obtained from the difference between ' $b$ ' and ' $c$ '.

With regard to the experimental results for quantifying the robustness of the technique to the uncertainties in the system 
natural frequency and damping ratio, the experimental results corresponding to the simulation results in Figures 4 and 5 are presented in Figures 10 and 11, respectively. It is apparent that the robustness results are also in good agreement.

It must be noticed in the simulation and experimental results that even when there is an error of as high as $\pm 35 \%$ in the natural frequency $\omega_{n}$ of the system, the amplitude of the residual vibration is still negligibly small. Further, it is apparent that any error in the damping ratio has negligibly small effect on the residual vibration reduction of the proposed input shaping method. This agrees with the fact that as the proposed method is very effective in reducing or eliminating the residual vibration, no vibration is remained to quantify the effect of the damping ratio on the amplitude of the residual vibration after the termination of the motion of the link. It must also be noted as the travel time is increased, the robustness of the technique to the natural frequency uncertainties increase significantly, compare the top and bottom plots of Figures 4, 5, 10, and 11. When the travel time is below the natural period of the system, i.e., $\tau_{t}=1.5 \pi / \omega_{n}$, width of the white areas in the top plots of the frequency ratio axis of Figures 4, 5, 10, and 11 is shorter than that of the corresponding middle and bottom plots. Another observation from the robustness results is that when the travel distances are calculated using Eq. 4, the robustness of the method to the natural frequency uncertainties increases significantly, as seen in Figures 4 and 5, and Figures 10 and 11.

Please recall from Section 3 that while the robustness of the first method, which is superimposition based input shaping, is limited, the robustness of the second method, which is convolution based input shaping, depends on the number of impulses. ${ }^{10}$ With this in mind, the numerical and experimental results show that the hybrid input shaping method we propose is robust to natural frequency as well as damping ratio uncertainties, and is also effective in reducing residual vibration.

\section{CONCLUSIONS}

The simulation and experimental results show that it is possible to obtain a significantly reduced vibration at the end of a move by employing the method of superimposing a ramp function onto another function and then convolving the preshaped function with a sequence of two impulses. The robustness of the technique to the uncertainties in the system natural frequency and damping ratio is also evaluated. It has been proven that the technique is robust to the natural frequency uncertainties of as high as $\mp 35 \%$ from the ideal natural frequency value, and it is insensitive to the damping ratio uncertainties. The results presented allow us to suggest that the proposed technique is versatile enough to apply it to the motion design of any flexible jointed manipulation system making a point-to-point motion.

\section{References}

1. G. Alici, S. Kapucu and S. Bayseç, "Swing-Free Transportation of Suspended objects with Robot Manipulators", Robotica 17, part 5, 513-521 (1999).

2. G. Alici, S. Kapucu and S. Bayseç, "On Preshaped Reference Inputs to Reduce Swing of Suspended Objects Transported with Robot Manipulators", Mechatronics 10, 609-626 (2000).

3. D. M. Aspinwall, "Acceleration Profiles for Minimizing Residual Response", ASME Journal of Dynamic Systems, Measurement, and Control 102, 3-6 (1980).

4. Cucciuo, R. Garziera and P. Righettini, "Vibration Control Input-Laws in Point to Point Motion: Theory and Experiments", Mechanisms and Machine Theory 33(4), 341349 (1998).

5. P. H. Meckl and W. P. Seering, "Experimental Evaluation of Shaped Inputs to Reduce Vibration for a Cartesian Robot", ASME Journal of Dynamic Systems, Measurement, and Control 112, 159-165 (1990).

6. G. Mimmi and P. Pennacchi, "Pre-shaping Motion Input for a Rotating Flexible Link", International Journal of Solids and Structures 38, 2009-2023 (2001).

7. B. R. Murphy and I. Watanable, "Digital Shaping Filters for Reducing Machine Vibration", IEEE Transactions on Robotics and Automation 8(2), 285-289 (1992).

8. M. N. Sahinkaya, "Input Shaping for Vibration-Free Positioning of flexible Systems", Proc. Instn. Mech. Engrs. 215(I), 467-481 (2001).

9. N. C. Singer and W. P. Seering, "Preshaping Command Inputs to Reduce System Vibration", ASME Journal of Dynamic Systems, Measurement, and Control 112, 76-82 (1990).

10. S. Kapucu, G. Alici and S. Baysec, "Residual Swing/Vibration Reduction Using a Hybrid Input Shaping Method", Mechanism and Machine Theory 36, 311-326 (2001).

11. S. Bayseç and J. R. Jones, "An Improved Model of an Electrohydraulic Servo Valve", International Proceedings of the Seventh IFToMM Congress, Sevilla, Spain (1987), pp. 1489-1494.

12. W. E. Singhose and N. C. Singer, "Effects of Input Shaping on Two Dimensional Trajectory Following", IEEE Transactions on Robotics and Automation, pp. 881-887, 12, No. 6, 881-887 (Dec., 1996).

13. W. Singhose, P. Porter, M. Kenison and E. Kriikku, "Effects of Hoisting on the Input Shaping Control of Gantry Cranes", Control Engineering Practice, A Journal of IFAC 8, 1159-1165 (2000).

14. P. H. Chang and J. Park, "A Concurrent Design of Input Shaping Technique and a Robust Control for High-Speed/HighPrecision Control of a Chip Mounter", Control Engineering Practice, A Journal of IFAC 9, 1279-1285 (2000).

15. M. W. Spong, "Modelling and Control of Elastic Joint Robots", Transaction of the ASME Journal of Dynamic Systems, Measurement, and Control 109, 310-319 (1987).

16. F. R. Steidel, An Introduction to Mechanical Vibrations (John Wiley and Sons, 1980). 